\title{
Aspects de la Conservation des Reptiles et des Amphibiens dans la Région de Daraina
}

\author{
Hery A. Rakotondravony
}

\begin{abstract}
This paper deals with conservation aspects of amphibians and reptiles in the Daraina region, north eastern part of Madagascar, where herpetological surveys undertaken between october 2002 and March 2003 and between October 2003 and March 2004 lead to the discovery of 36 amphibians and 74 reptiles. Thirteen taxa among them are currently known only within the Daraina forest areas, and 20 are listed in the CITES (Convention on International Trade in Endangered Species of Wild Fauna and Flora) Appendices II and I. These high herpetofaunal species diversity and endemism demonstrate the value of this site in terms of terrestrial vertebrate conservation in Madagascar, and the need of an urgent conservation strategy to protect these natural resources.
\end{abstract}

\section{INTRODUCTION}

L'importance des écosystèmes forestiers naturels malgaches dans le maintien de la biodiversité terrestre n'est plus à démontrer, considérant le niveau de diversité et du taux d'endémicité très importants à Madagascar (cf. Goodman and Benstead 2005). Mais en raison du taux de déforestation très élevé dans ce pays, ces habitats naturels sont parmi les plus menacés au monde. Actuellement, six pour cent des forêts naturels malgaches sont inclus dans le réseau national d'aires protégées (Du Puy and Moat 2003); et d'autres sites méritent également d'être conservés notamment en raison des menaces précitées. Mais à l'égard des limitations auxquelles fait face I'Etat Malgache dans la réalisation et le renforcement des politiques de législations forestières, il est évident que l'insertion d'un site parmi le réseau national d'aires protégées devrait suivre certaines logiques de priorités. Un des critères favorisant l'attribution de statuts légaux de protection pour ce site serait le niveau de diversité et d'endémisme qui est significativement important au moins pour un groupe d'organismes donné. Ce document essaie de démontrer l'importance de la région de Daraina en terme de conservation en abritant des niveaux importants de diversité et d'endémisme en reptiles et amphibiens.

\footnotetext{
MÉTHODOLOGIE

RÉGION D'ÉTUDES La ville de Daraina (S 1312'; E $049^{\circ} 39^{\prime}$ )

se situe dans la province d'Antsiranana, environ à mi-chemin entre Ambilobe et Vohémar. La région communément appelée «région de Daraina» se trouve à l'extrême Nord-Est de Madagas-
}

Département de Biologie Animale, Faculté des Sciences, Université d'Antananarivo, BP 906 et Ecology Training Program, WWF, BP 738 Antsakaviro, Antananarivo (101).

E-mail: etp@wwf.mg

car, et est délimitée naturellement par les fleuves Loky au nord et Manambato au sud (Figure 1). L'altitude varie de 0 à 1,170 m. La température moyenne annuelle serait de $26^{\circ} \mathrm{C}$ et la pluviométrie annuelle serait de 1,280 mm (Rakotondravony données nonpubl.).

Les écosystèmes forestiers naturels de cette région se présentent sous forme de fragments et blocs de forêts en mosaïques dans une échelle microgéographique de $44 \mathrm{~km}^{2}$. Elle se situe dans un carrefour bioclimatique important (Cornet 1974); et avec ses structures pédologiques très complexes (Besairie 1965), elle possède différents types de formations végétales. Ces dernières varient à partir de forêts caducifoliées en dessous de $350 \mathrm{~m}$ d'altitude aux forêts humides semi-sempervirentes à plus de $700 \mathrm{~m}$, avec diverses formations de transition dans les étages altitudinaux intermédiaires.

La région de Daraina a été identifiée par Conservation International (1995) comme une des priorités en matières de conservation à Madagascar, surtout en raison de l'existence d'une espèce de primate qui y est endémique (Indridae). L'arrêté ministériel n 5862/05-MINENVEF du 31 mai 2005 portant création de la Station Forestière à Usage Multiple de Loky-Manambato (70'837 ha) a conduit à l'inclusion de la majeure partie des plus grands blocs forestiers de la région parmi le réseau national d'aires protégées malgaches. La nouvelle aire protégée comprend les sept blocs forestiers les plus importants de la région de Daraina en terme de superficie, Ambohitsitondroina, Ampondrabe, Antsahabe, Antsaharaingy, Bekaraoka, Binara, Bobankora et la forêt littorale de Sahaka.

COLLECTE DES DONNÉES Des séries d'inventaires d'am-

phibiens et de reptiles ont été conduites dans certains blocs et fragments forestiers de la région de Daraina entre octobre 2002 et mars 2003, et entre octobre 2003 et mars 2004 (tableau 1). Ces mois correspondent aux saisons chaudes et pluvieuses d'activités maximales des amphibiens et des reptiles, favorables à leurs recensements. L'équipe a été composée de l'auteur et d'un assistant de terrain. Trois principales méthodes ont été utilisées: 1) observation directe le long d'itinéraires échantillons; 2) fouille systématique des lieux de refuges et 3) piégeage par trous-pièges («pitfall») avec barrière plastique. Des descriptions de ces techniques sont données dans Raxworthy et al. (1998) et Raselimanana et al. (2000).

\section{RÉSULTATS ET DISCUSSION}

RICHESSE SPÉCIFIQUE Un total de 110 taxons herpétologiques a été recensé dans douze massifs forestiers de la 


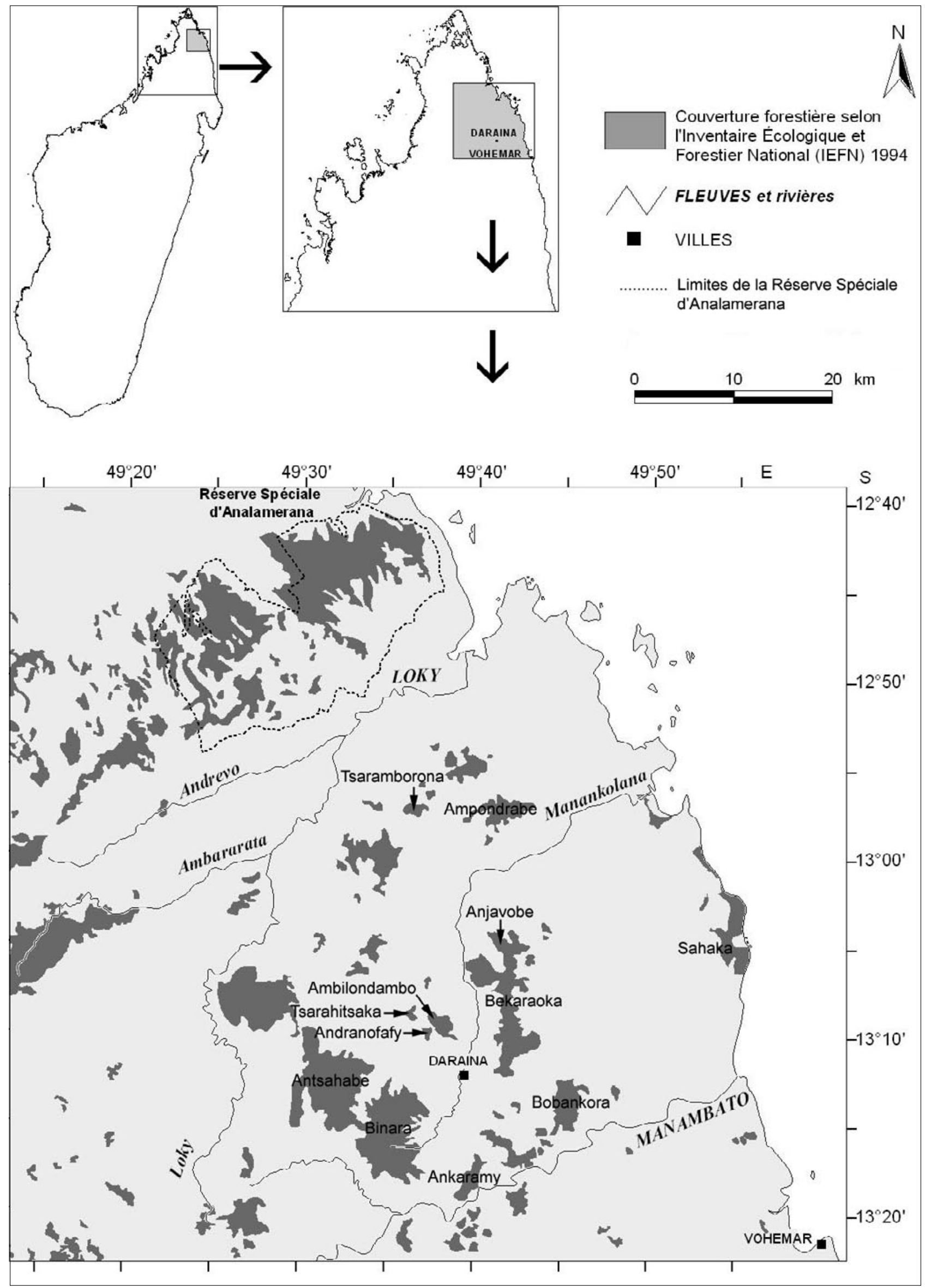

FIGURE 1. Carte montrant les différents blocs et fragments forestiers de la région de Daraina, d'après I'Inventaire Ecologique Forestier National (IEFN), 1994. Cette carte montre que le fragment forestier d'Anjavobe était rattaché au massif de Bekaraoka il y a quelques années mais lors de nos travaux sur le terrain, Anjavobe est actuellement isolé de ce massif. Au nord de la région se trouve la Réserve Spéciales d'Analamerana. 
TABLEAU 1. Noms, coordonnées géographiques, superficie, altitude, dates d'inventaire et richesses spécifiques en amphibiens et reptiles des massifs forestiers de Daraina concernés par cette étude.

\begin{tabular}{|c|c|c|c|c|c|c|}
\hline $\begin{array}{l}\text { SITES } \\
\text { (SUPERFICIE [ha]; PRESSIONS } \\
\text { ANTHROPIQUES }{ }^{1} \text { ) }\end{array}$ & LATITUDE & LONGITUDE & ALTITUDE (M) & DATE D'INVENTAIRE & REPTILES & AMPHIBIENS \\
\hline Ambilondambo (319; BD, CSB, PZ) & $\mathrm{S} 13^{\circ} 09,7^{\prime}$ & E $049^{\circ} 38,7^{\prime}$ & $250-540$ & 20-28 jan. 2003 & 28 & 7 \\
\hline Ampasibe $(67 ; \mathrm{CSB}, \mathrm{PZ})$ & $S 13^{\circ} 09,9^{\prime}$ & E $049^{\circ} 37,8$ & $230-410$ & 01-07 mar. 2004 & 21 & 5 \\
\hline Ampondrabe (1231;BD, PZ) & S $12^{\circ} 58,4^{\prime}$ & $\mathrm{E} 049^{\circ} 42,2^{\prime}$ & $80-580$ & 10 nov. -23 nov. 2003 & 31 & 7 \\
\hline Anjavobe (560; BD, PZ, CSB) & $\mathrm{S} 13^{\circ} 04,0^{\prime}$ & $\mathrm{E} 049^{\circ} 41,4^{\prime}$ & $90-420$ & 26 nov.-02 déc. 2002 & 22 & 5 \\
\hline Ankaramy (472; CSB, PZ, OR) & S $13^{\circ} 17,0^{\prime}$ & $\mathrm{E} 049^{\circ} 40,8^{\prime}$ & $160-360$ & 29 nov.-05 déc. 2003 & 25 & 9 \\
\hline \multirow[t]{2}{*}{ Antsahabe (3404; CSB, PZ) } & S $13^{\circ} 12,6^{\prime}$ & E $049^{\circ} 33,8^{\prime}$ & $350-500$ & 16-23 oct. 2003 & 39 & 17 \\
\hline & $S 13^{\circ} 12,6^{\prime}$ & E $049^{\circ} 33,5^{\prime}$ & $450-950$ & 23-30 oct. 2003 & & \\
\hline \multirow[t]{4}{*}{ Bekaraoka (4150; BD, OR, CSB, PZ) } & $\mathrm{S} 13^{\circ} 09,9^{\prime}$ & E $049^{\circ} 43,0^{\prime}$ & 180-330 & 04-11 déc. 2003 & 37 & 11 \\
\hline & S $13^{\circ} 11,7^{\prime}$ & $\mathrm{E} 049^{\circ} 42,6^{\prime}$ & $170-310$ & 06-13 fév. 2003 & & \\
\hline & $S 13^{\circ} 10,6^{\prime}$ & $\mathrm{E} 049^{\circ} 42,0^{\prime}$ & $150-340$ & 07-14 déc. 2003 & & \\
\hline & $\mathrm{S} 13^{\circ} 06,3^{\prime}$ & $\mathrm{E} 049^{\circ} 42,7^{\prime}$ & $110-360$ & 18-25 nov. 2002 & & \\
\hline \multirow[t]{4}{*}{ Binara (4143; CSB, BD, PZ) } & S $13^{\circ} 15,7^{\prime}$ & E $049^{\circ} 36,4^{\prime}$ & $610-1070$ & 24-31 oct. 2002 & 53 & 25 \\
\hline & S $13^{\circ} 15,2^{\prime}$ & E $049^{\circ} 35,5^{\prime}$ & $710-1170$ & 31 oct. -07 nov. 2002 & & \\
\hline & $S 13^{\circ} 14,4^{\prime}$ & E $049^{\circ} 34,8^{\prime}$ & $710-1100$ & 07-14 nov. 2002 & & \\
\hline & $\mathrm{S} 13^{\circ} 14,3^{\prime}$ & E $049^{\circ} 37,5^{\prime}$ & $210-550$ & $\begin{array}{l}\text { 16-24 oct. } 2002 \text { et } \\
15-21 \text { fév. } 2004\end{array}$ & & \\
\hline \multirow[t]{3}{*}{ Bobankora (1121;BD, PZ, CSB) } & S $13^{\circ} 13,4^{\prime}$ & E $049^{\circ} 45,6^{\prime}$ & $350-610$ & 20-27 fév. 2003 & 40 & 13 \\
\hline & S $13^{\circ} 13,6^{\prime}$ & $\mathrm{E} 049^{\circ} 45,1^{\prime}$ & $140-350$ & 13-20 fév. 2003 & & \\
\hline & S $13^{\circ} 12,7^{\prime}$ & $\mathrm{E} 049^{\circ} 46,3^{\prime}$ & $90-350$ & 28 fév-07 mar. 2003 & & \\
\hline Sahaka (2678; CSB, BD, PZ) & $\mathrm{S} 13^{\circ} 04,7^{\prime}$ & E $049^{\circ} 54,1^{\prime}$ & $10-50$ & 23-28 fév. 2004 & 21 & 5 \\
\hline Tsarahitsaka (47;CSB, PZ, BD) & $\mathrm{S} 13^{\circ} 08,9^{\prime}$ & $\mathrm{E} 049^{\circ} 37,4^{\prime}$ & $230-430$ & 28 jan.-04 fév. 2003 & 25 & 6 \\
\hline Tsaramborona (216; CSB, PZ, BD) & S $12^{\circ} 57,4^{\prime}$ & E $049^{\circ} 36,8^{\prime}$ & $150-450$ & 04-10 nov. 2003 & 21 & 0 \\
\hline
\end{tabular}

${ }^{1} \mathrm{BD}=$ coupe de bois durs, $\mathrm{CSB}=$ culture sur brûlis, $\mathrm{OR}=$ orpaillage, $\mathrm{PZ}=$ pâturage de zébus.

région de Daraina. La liste complète de ces taxons est accessible via l'auteur. La faune herpétologique de ces forêts est caractérisée par le nombre élevé en espèces reptiliennes, une des signes de l'aridité prononcée dans la région. Les amphibiens représentaient $32.7 \%$ (36 espèces) de cette faune, tandis que les 74 espèces restantes (67.3\%) étaient des reptiles. Ce niveau de diversité herpétologique élevé témoigne l'importance de la place tenue par cette région en terme de conservation de la diversité des vertébrés terrestres malgache. Apparemment, peu nombreux sont les sites de la Grande île ayant des richesses spécifiques en reptiles et amphibiens plus élevées que Daraina. Par exemple, la Réserve Spéciale (RS) de Manongarivo et le Parc National (PN) de la Montagne d'Ambre abritent respectivement 86 et 70 espèces (Rakotomalala 2002; Raxworthy and Nussbaum 1994); tandis que Nosy Be et les îles voisines en abritent 81 (Andreone et al. 2003). Raxworthy et al.(1998) mentionnent 93 espèces pour la RS d'Anjanaharibe-Sud. Dans l'état des connaissances actuelles, le seul site qui possède une diversité herpétologique avoisinant celle de Daraina serait le PN de Marojejy (113 espèces, Raselimanana et al. 2000). Néanmoins, ces comparaisons devraient être traitées avec réserves puisque les efforts déployés dans chacun de ces sites ont été très inégaux, malgré les analogies sur les plans méthodologiques lors des inventaires.

ESPÈCES D'AMPHIBIENS ET DE REPTILES MENACÉES

DANS LA RÉGION DE DARAINA La région abrite plusieurs espèces inscrites aux Annexes de la CITES (Convention on International Trade in Endangered Species of Wild Fauna and Flora) dont deux en Annexe I (Acrantophis madagascariensis et
Sanzinia madagascariensis, Boidae, Reptilia) et 20 en Annexe II (Amphibia- Mantellidae - Mantella spp.: deux espèces; Reptilia - Chamaeleonidae - Brookesia spp.: quatre espèces, Calumma spp.: deux espèces et Furcifer spp.: quatre espèces; Gekkonidae - Phelsuma spp.: quatre espèces et Uroplatus spp.: quatre espèces). Elles constituent $20 \%$ des espèces d'amphibiens et de reptiles recensées lors de cette étude, ce qui est une proportion non négligeable. Toutefois, aucune collecte de ces espèces à des fins commerciaux n'a été signalée lors des travaux effectués dans la région. La principale menace pour ces espèces est la destruction de leurs habitats naturels par les pressions anthropiques (voir Tableau 1). Il est ainsi évident que la gestion durable de ces espèces dans cette région se situerait dans I'application d'une meilleure gestion de leurs habitats naturels qui sont primordialement les forêts primaires.

IMPORTANCE DE LA DIVERSITÉ DES MILIEUX NATURELS

SUR LES RICHESSES SPÉCIFIQUES Nombreux auteurS ont souligné l'importance des grands blocs forestiers dans le maintien de la diversité biologique aussi bien à Madagascar qu'ailleurs. Cependant, une analyse des communautés d'amphibiens et de reptiles des massifs forestiers de la région a montré que la superficie y jouerait un rôle "secondaire» dans la distribution des richesses spécifiques; le facteur le plus important semble être la diversité des habitats naturels engendrés par l'altitude (Rakotondravony, données nonpubl.). En outre, les études effectuées dans cette région mettent en évidence l'existence d'une zonation altitudinale des communautés d'amphibiens et de reptiles dans la région, ce qui signifie l'existence de changements de compositions des communau- 
tés d'amphibiens et de reptiles le long du gradient altitudinal. Ce fait joue un rôle important, en y engendrant des niveaux de diversités plus élevés pour les massifs montagneux les plus importants.

IDENTIFICATIONS DES MASSIFS FORESTIERS PRIORI-

TAIRES EN MATIÈRES DE CONSERVATIONS La diversité régionale de Daraina semble être bien représentée dans les massifs forestiers de Binara, d'Antsahabe et de Bobankora. II est à souligner que ce sont ces trois massifs qui possèdent la diversité d'habitats naturels les plus importants dans la région, en raison de l'oscillation altitudinale plus élevée que I'on y rencontre. Ensemble, les massifs forestiers d'Antsahabe, de Binara et de Bobankora arrivent à conserver $94.4 \%$ des espèces d'amphibiens et $86.5 \%$ des espèces de reptiles forestiers de la région de Daraina (Rakotondravony, données nonpubl.). A première vue, ces massifs de superficies importantes sont les prioritaires en matières de conservation de reptiles et d'amphibiens dans la région de Daraina. Cependant, certaines espèces d'amphibiens et de reptiles n'ont pas été enregistrées dans l'ensemble formé par les trois massifs forestiers les plus diversifiés en reptiles et en amphibiens. II s'agit de trois espèces d'amphibiens et de 10 espèces de reptiles. La présence de ces espèces semble indiquer l'importance des autres massifs forestiers de moindre superficie dans le maintien de la diversité biologique dans la région.

ESPÈCES SUGGÉRÉES ENDÉMIQUES À LA RÉGION DE

DARAINA Les forêts de Daraina possèdent une proportion significative de son herpétofaune qui pourrait y être endémique. II s'agit de 13 espèces dont I'identification n'a pas pu être établie avec certitude et qui pourraient constituer des formes nouvelles pour la science. Elles constituent $12 \%$ environ de I'herpétofaune de la région de Daraina, un taux qui n'est pas le moindre à l'instar de ceux observés dans d'autres sites de Madagascar: $12 \%$ environ pour le PN du Marojejy (Raselimanana et al. 2000) et $13 \%$ pour le PN d'Andringitra (Raxworthy and Nussbaum 1996).

\section{CONCLUSION}

Les niveaux de la diversité et d'endémisme importants (rivalisant ceux des autres sites malgaches protégés) confirment l'importance de la région de Daraina dans le maintien de la biodiversité. Ces faits témoignent également que les forêts de la région de Daraina tiennent une place importante parmi le réseau national d'aires protégées de Madagascar. Néanmoins, $20 \%$ des espèces herpétofauniques recensées lors des études sur le terrain sont inscrites aux Annexes I et II de la CITES. La gestion durable de ces ressources naturelles réside dans I'application d'une meilleure gestion de l'ensemble des écosystèmes forestiers dans cette région.

\section{REMERCIEMENTS}

Mes vifs remerciements reviennent au Pr. Steve Goodman pour ses commentaires sur la version préliminaire de ce document. Mes gratitudes vont également aux populations de la région de Daraina pour leurs hospitalités lors de mes séjours dans cette région. La carte a été fournie gracieusement par Lucienne Wilmé. Cette étude fait partie intégrante de l'Ecology Training Program de WWF avec financement de CEPF à travers I'ONG Fanamby et de Conservation International. L'ONG Fanamby a assuré en majeure partie les moyens logistiques.

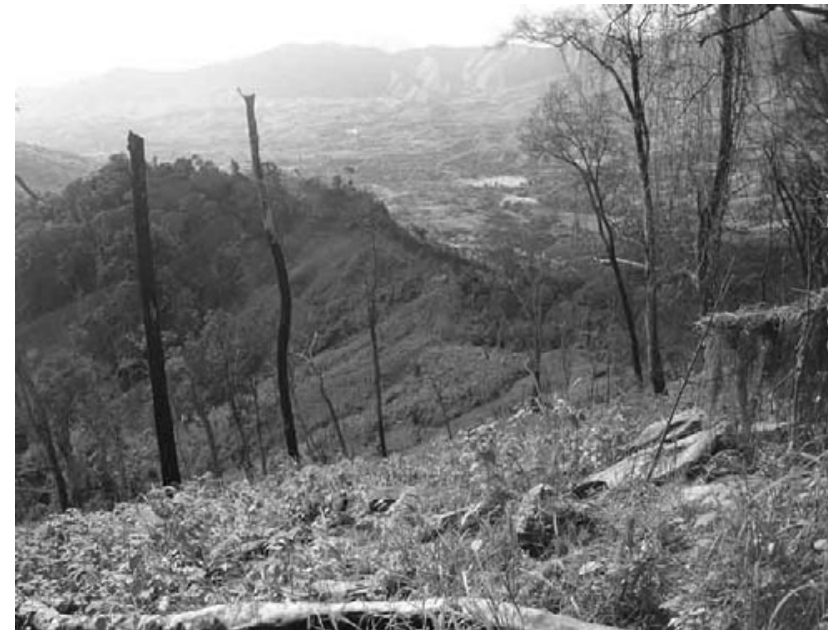

FIGURE 2. Destruction des forêts naturelles dans le massif de Binara, au sudouest de Daraina.

\section{REFERENCES}

Andreone, F., Glaw, F., Nussbaum, R.A., Raxworthy, C.J., Vences, M. and Randrianirina, J.E. 2003. The amphibians and reptiles of Nosy Be (NW Madagascar) and nearby islands: a case study of diversity and conservation of an insular fauna. Journal of Natural History 37, 17: 2119-2149

Besairie, H. 1965. Esquisse géologique et lithographique de Madagascar. In: Notice de la Carte de Madagascar, H. Humbert and G. Cours Darne (eds.), pp. 14-18. Travaux de la Section Scientifique et Technique de I'Institut Français de Pondichéry, hors série 6

Conservation International. 1995. Priorités de Conservation de la Diversité Biologique à Madagascar. ONE. Direction des Eaux et Forêts. ANGAP. PNUD. USAID-Madagascar. [CD-ROM].

Cornet, A. 1974. Essai de cartographie bioclimatique à Madagascar. Notice Explicative de I'ORSTOM 55: 1-38

Du Puy, D.J., and Moat, J. 2003. Using geological substrate to identify and map primary vegetation types in Madagascar and the implications for planning biodiversity conservation. In: The Natural History of Madagascar, S.M. Goodman and J.P. Benstead (eds.), pp 51-67. The University of Chicago Press, Chicago.

Goodman, S.M. and Benstead, J.P. 2005. Updated estimates of biotic diversity and endemism for Madagascar. Oryx 39, 1: 1-5.

Rakotomalala, D. 2002. Diversité des reptiles et amphibiens de la Réserve spéciale de Manongarivo, Madagascar. In: Inventaire Floristique et Faunistique de la Réserve Spéciale de Manongarivo (NW Madagascar), L. Gautier and S.M. Goodman (eds.), pp 339-359. Boissiera 59

Raselimanana, A.P., Raxworthy, C.J. and Nussbaum, R.A. 2000. Herpetofaunal species diversity and elevational distribution within the Parc National de Marojejy, Madagascar. In: A Floral and Faunal Inventory of the Parc National de Marojejy, Madagascar: With Reference to Elevational Distribution, S.M. Goodman (ed.), pp 157-174. Fieldiana: Zoology, new series 97

Raxworthy, C.J. and Nussbaum, R.A. 1994. A rainforest survey of amphibians, reptiles and small mammals at Montagne d'Ambre, Madagascar. Biological Conservation 69: 65-73

RAXWORTHY, C.J. AND NUSSBAUM, R.A. 1996. Amphibians and reptiles of the Réserve Naturelle Intégrale d'Andringitra, Madagascar: A study of elevational distribution and local endemicity. In: A Floral and Faunal Inventory of the Eastern Slopes of the Réserve Naturelle d'Andringitra, Madagascar: With Reference to Elevational Variation, Goodman, S.M. (ed), pp 158-170. Fieldiana: Zoology, new series 85

Raxworthy, C.J., Andreone, F., Nussbaum, R.A., Rabibisoa, N. and Randriamahazo, H. 1998. Amphibians and reptiles of the AnjanaharibeSud massif, Madagascar: Elevational distribution and regional endemicity. In: A Floral and Faunal Inventory of the Eastern Slopes of the Réserve Spéciale d'Anjanaharibe-Sud: With Reference to Elevational Variation, S.M. Goodman (ed.), pp 79-92. Fieldiana: Zoology, new series 90. 